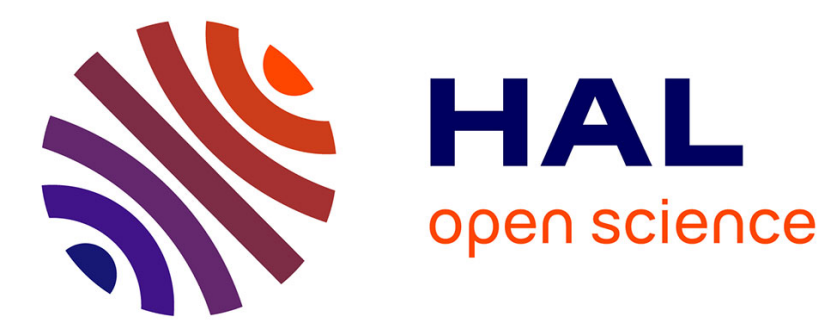

\title{
Petits entrepreneurs étrangers en ville. Claire Zalc
}

\section{To cite this version:}

Claire Zalc. Petits entrepreneurs étrangers en ville.: Localisations urbaines, réseaux migratoires et solidarités professionnelles dans la Seine pendant l'entre-deux-guerres. Histoire urbaine, 2001, n 4 (2), pp.67. 10.3917/rhu.004.0067 . hal-03416527

\section{HAL Id: hal-03416527 \\ https://hal.science/hal-03416527}

Submitted on 5 Nov 2021

HAL is a multi-disciplinary open access archive for the deposit and dissemination of scientific research documents, whether they are published or not. The documents may come from teaching and research institutions in France or abroad, or from public or private research centers.
L'archive ouverte pluridisciplinaire HAL, est destinée au dépôt et à la diffusion de documents scientifiques de niveau recherche, publiés ou non, émanant des établissements d'enseignement et de recherche français ou étrangers, des laboratoires publics ou privés. 


\section{PETITS ENTREPRENEURS ÉTRANGERS EN VILLE.}

Localisations urbaines, réseaux migratoires et solidarités professionnelles dans la Seine pendant l'entre-deux-guerres

\section{Claire Zalc}

Société française d'histoire urbaine | « Histoire urbaine »

$2001 / 2 \mathrm{n}^{\circ} 4 \mid$ pages 67 à 82

ISSN 1628-0482

ISBN 291435004x

DOI 10.3917/rhu.004.0067

Article disponible en ligne à l'adresse :

https://www.cairn.info/revue-histoire-urbaine-2001-2-page-67.htm

Distribution électronique Cairn.info pour Société française d'histoire urbaine.

(C) Société française d'histoire urbaine. Tous droits réservés pour tous pays.

La reproduction ou représentation de cet article, notamment par photocopie, n'est autorisée que dans les limites des conditions générales d'utilisation du site ou, le cas échéant, des conditions générales de la licence souscrite par votre établissement. Toute autre reproduction ou représentation, en tout ou partie, sous quelque forme et de quelque manière que ce soit, est interdite sauf accord préalable et écrit de l'éditeur, en dehors des cas prévus par la législation en vigueur en France. Il est précisé que son stockage dans une base de données est également interdit. 


\section{Petits entrepreneurs étrangers en ville.}

\section{Localisations urbaines, réseaux migratoires et solidarités professionnelles dans la Seine pendant l'entre-deux-guerres}

D our qui ambitionne de travailler sur l'histoire des solidarités profesP sionnelles en ville, il convient, d'emblée, de réfléchir concrètement à deux problèmes méthodologiques essentiels de l'histoire urbaine: d'une part, la question de la pertinence des échelles adoptées pour étudier les phénomènes urbains dans le passé et, d'autre part, la question du rôle des acteurs économiques dans le tracé des formes de la ville ${ }^{1}$. Lors de l'étude des implantations des petits artisans et commerçants étrangers de l'agglomération parisienne dans l'entre-deux-guerres, ces questionnements ont trouvé un terrain doublement spécifique en ce qu'il traite à la fois de l'immigration et de la petite entreprise, deux objets qui posent, de manière centrale, la question du rapport aux structures spatiales. À une époque qui voit la genèse des "quartiers d'immigration" de la capitale (expression qui désigne autant des zones de forte visibilité que des espaces de concentration des immigrants), l'analyse du rôle des indépendants dans la formation et l'organisation sociale de ces quartiers permet de mieux comprendre comment se déterminent les logiques d'implantation professionnelle des migrants et de mesurer leur stabilité dans le temps. En effet, les étrangers ne se répartissent pas de manière homogène dans l'espace parisien. Quels sont les critères qui déterminent leur quartier d'implantation et leurs parcours dans l'espace? L'étude des petites entreprises tenues par des étrangers contribue à éclairer cette question. En effet, l'activité économique réalisée dans le cadre d'un atelier ou d'une boutique

1. Ce texte est issu des réflexions développées avec l'ensemble des participants de l'atelier "Solidarités professionnelles, réseaux citadins, mutations spatiales: les formes d'exercice des métiers en ville du $\mathrm{xvI}^{\mathrm{e}}$ au $\mathrm{xx}^{\mathrm{e}}$ siècles» organisé à Berlin le 31 août 2000 par Natacha Coquery et Caroline Varlet, dans le cadre de la $5^{\mathrm{e}}$ Conférence internationale d'histoire urbaine. 
engendre un rapport particulier au territoire: les petites entreprises tenues par des migrants sont fondées sur des liens d'interconnaissance. De plus, elles suscitent des pratiques de sociabilités spécifiques.

Le département de la Seine offre un cadre d'étude idéal: il constitue, au lendemain de la Grande Guerre, une destination privilégiée par les migrants étrangers, dont le nombre passe de 228000 à 449000 entre 1921 et 1931. De plus, il concentre l'installation des populations en marge des grands flux migratoires à destination de la France à cette période, en grande majorité formés, faut-il le rappeler, de travailleurs salariés de la grande industrie ${ }^{2}$. Dès 1921 , plus du quart des actifs étrangers $(28,5 \%)$ recensés dans le département de la Seine n'ont pas le statut de salariés, qu'ils soient comptabilisés parmi les "patrons» ou les "travailleurs isolés ${ }^{3}$. Enfin, le département de la Seine offre au chercheur la possibilité de croiser des sources originales pour aborder la question: sources administrative (registre du commerce), démographique (listes nominatives du recensement), fiscale (rôles d'impositions des patentes) et biographique (récits de vie) permettent de mener conjointement l'analyse des trajectoires des petits entrepreneurs étrangers (biographiques, professionnelles et urbaines) et celle des configurations relationnelles dans lesquelles ils s'insèrent.

Il s'agit alors de réfléchir sur la nature des liens entre parcours migratoires, parcours professionnels et trajets dans la ville, en travaillant sur l'idée de réseau migratoire. La confrontation entre les résultats de l'analyse selon les différentes échelles considérées amène, tout d'abord, à montrer la pluralité des logiques déterminant la localisation des petits artisans et commerçants migrants. Puis, en déplaçant le point de vue sur les stratégies des acteurs, on tentera d'évaluer les formes sociales des implantations professionnelles en évoquant le fonctionnement des filières migratoires pour comprendre comment les liens d'interconnaissance noués entre ces individus dessinent des filières professionnelles autonomes, complémentaires ou concurrentes.

\section{Implantations urbaines: à chaque échelle, son réseau}

Plusieurs types d'observations peuvent être menés afin de décrire les implantations commerciales et artisanales des étrangers dans l'agglomé-

2. Voir Gérard Noiriel, Le creuset français, Histoire de l'immigration $\mathrm{XIX}^{e}-\mathrm{XX} \mathrm{X}^{e}$ siècle, Paris, Le Seuil, 1988 ; Janine Ponty, Polonais méconnus. Histoire des travailleurs immigrés dans l'entre-deuxguerres, Paris, Publications de la Sorbonne, 1990.

3. Statistique générale de la France, Résultats statistiques du recensement général de la population de 1921, Paris, Imprimerie nationale, vol. 3. 
ration parisienne. La première, établie à partir d'un échantillon de 2500 immatriculations effectuées entre 1920 et 1939 au registre du commerce de la Seine, constitue une approche statistique relativement large qui permet d'esquisser les grands traits des localisations dans l'agglomération en se focalisant sur les phénomènes de concentration. La seconde, qui adopte les dimensions plus réduites des quartiers, voire des rues de la capitale, à partir du dépouillement des listes nominatives des recensements et des rôles d'imposition des patentes, invite plutôt à prendre en considération les différents types de solidarités à l'œuvre dans le fonctionnement économique des espaces parisiens ${ }^{4}$.

\section{A L'échelle du département de la Seine, LA PERTINENCE DE L'INDICATEUR MIGRATOIRE}

Alors que la banlieue connaît une forte croissance démographique dans l'entre-deux-guerres, Paris reste la localisation préférée par les deux tiers des petits entrepreneurs du département. Les étrangers sont les plus nombreux, relativement, à déclarer une adresse professionnelle dans la capitale: $81 \%$ pour $70 \%$ des Français. L'étude de la part des établissements ouverts en banlieue selon la nationalité de l'entrepreneur montre des différences sensibles entre immigrants. En effet, si 36\% des Italiens immatriculent un établissement en banlieue entre 1922 et 1939, $12 \%$ des petits entrepreneurs russes choisissent une installation à l'extérieur des fortifs et seulement $2 \%$ des Polonais.

On peut ainsi distinguer, parmi les petits entrepreneurs migrants, deux types de trajectoires : ceux, tout d'abord, pour lesquels le statut d'indépendant vient, après plusieurs années passés en France, sanctionner la réussite d'un itinéraire professionnel effectué, la plupart du temps, comme salarié, et pour lesquels l'indépendance économique consacre une mobilité socioprofessionnelle ascendante - c'est le cas des Belges, des Suisses ou encore de certains Italiens arrivés en France dès les années 1880-1890 -; puis, un certain nombre de migrants, originaires de "jeunes» régions d'immigration qui tiennent le rôle de pionniers de filières en constitution. Le petit commerce et l'artisanat représentent alors un moyen de trouver rapidement un revenu qui, éventuellement, permet d'activer la filière migratoire. Les premiers, qui sont pour une large majorité déjà installés dans la région parisienne, ne rechignent pas à s'établir en banlieue, terre souvent fami- 
lière puisque ils ont souvent, précédemment, été employés par les entreprises de la grande industrie qui prospèrent dans les communes de la périphérie ${ }^{5}$. Quant aux pionniers migrants, ils semblent au contraire privilégier un établissement au sein des limites de la capitale, puisque dans leur majorité, les étrangers nouvellement arrivés dans la région parisienne s'installent à Paris intra muros.

La même pertinence de la chronologie migratoire dans les logiques d'implantation des commerçants et artisans nés en province, plus nombreux que la moyenne à s'établir en banlieue $(32 \%$ contre $28 \%$ des commerçants originaires du département de la Seine) est constatée. En effet, parmi les provinciaux $42 \%$ des originaires de l'Aveyron, lieu de départ de flux migratoires anciens et structurés vers l'agglomération parisienne, s'installent en banlieue contre $13 \%$ des petits entrepreneurs nés en Saône et Loire ${ }^{6}$. Il semble que ce ne soit pas la nature, interrégionale ou internationale, de la migration qui explique la différenciation spatiale des petits commerces dans l'agglomération, mais plutôt la chronologie de la migration et le moment où prend place, dans l'itinéraire biographique, l'ouverture d'une petite entreprise. Le départ vers la banlieue semble, en effet, plutôt s'effectuer après quelques temps passés dans la capitale comme en témoigne l'âge plus élevé des petits entrepreneurs de banlieue à leur installation: 38,5 ans contre 37 ans en moyenne. Ces données permettent d'établir l'influence de l'itinéraire migratoire et professionnel des petits entrepreneurs sur leur répartition dans le département de la Seine que confirme l'observation des implantations commerciales dans la capitale.

\section{A L'ÉCHELle DE LA Ville, DES LOGIQUES DE SUCCESSION ENTRE MIGRANTS}

Si l'on concentre le regard sur l'espace enceint par les fortifs, on observe les différences de répartition des petits entreprises tenues par des migrants entre les arrondissements parisiens. En effet, les boutiques et les ateliers des provinciaux sont sensiblement sur-représentés à l'Ouest, alors que les commerçants et les artisans nés à l'étranger choisissent plutôt les arrondissements du Nord et de l'Est. Plus précisément, on constate que ces derniers s'installent dans le centre de Paris (notamment dans les $2^{\mathrm{e}}, 3^{\mathrm{e}}$ et

5. Ainsi des Russes à Boulogne-Billancourt comme le montre Olivier Le Guillou, «L'émigration russe en France, Boulogne-Billancourt et les usines Renault: lieux d'habitation et emplois des émigrés russes dans l'entre-deux-guerres » dans Éric Guichard et Gérard Noiriel (sous la direction de), Construction des nationalités et immigration dans la France contemporaine, Paris, Presses de l'ENS, 1997, p. 215-257.

6. Françoise Raison-Jourde, La colonie auvergnate de Paris au $\mathrm{XIX}^{e}$ siècle, Paris, Commission des travaux historiques de la ville de Paris, $\mathrm{n}^{\circ} 14,1976$. 
$4^{\mathrm{e}}$ arrondissements), quand les provinciaux sont plus attirés par les arrondissements périphériques ${ }^{7}$. À la fin du XIX ${ }^{\mathrm{e}}$ siècle, les Auvergnats avaient pourtant leurs cafés dans le centre et l'est de la capitale et dans les mêmes arrondissements où les étrangers s'établissent dans l'entre-deux-guerres ${ }^{8}$. Il semble ainsi que certains quartiers se définissent comme des territoires d'accueil des "nouveaux migrants", alors que d'autres constituent des territoires de seconde installation. Certes, le facteur économique explique, pour une large part, la concentration des migrants arrivés de fraîche date: la moindre cherté des baux et des loyers constitue, sans nul doute, une raison primordiale à l'attrait de certains quartiers de la capitale comme Belleville, Clignancourt ou encore La Villette.

Mais il faut également inscrire le choix d'une localisation au sein d'un itinéraire déterminé par des réseaux professionnels et des filières migratoires. L'École de Chicago, dès les années 1920, a montré que les regroupements de populations tenaient autant de l'avancée dans le parcours migratoire que de la réussite professionnelle ${ }^{9}$. L'installation à son compte s'inscrit dans un parcours; elle n'est pas simplement déterminée par une logique économique. Les récits de vie font ainsi état des mécanismes à l'œuvre dans le choix d'une implantation: lorsque Maurice Arnoult, né à Bagneaux-sur-Loing, en Seine-et-Marne, arrive à Paris, à l'âge de 14 ans, il a en poche un contrat d'apprentissage chez un ami de la famille, cordonnier à Belleville. Initié au métier dans le quartier, il n'envisage pas d'autre localisation professionnelle lorsqu'il ouvre son propre atelier de chaussures, quelques années plus $\operatorname{tard}^{10}$. En effet, le statut de petit entrepreneur indépendant engage un certain nombre de caractéristiques en termes d'insertion socio-économique: nécessité d'un petit capital de départ, besoin d'un local professionnel accessible et surtout présence de réseaux de fournisseurs et de clients potentiels.

Belleville offre d'ailleurs l'exemple d'un quartier dont le paysage économique est profondément renouvelé par l'installation de petits entrepreneurs migrants entre les deux guerres.

7. Cf. les cartes établies par Judith Rainhorn et Claire Zalc, «Lieux du petit commerce italien à Paris dans l'entre-deux-guerres. Noria migratoire à La Villette», Villes, espaces et territoires, Travaux de l'EHESS 1999, Paris, 1999, p. 63.

8. F. Raison-Jourde, La colonie auvergnate..., op. cit., p. 191-212.

9. Robert Park et William Burgess, The City, Chicago, University of Chicago Press, 1925, (trad. fr. dans L'École de Chicago, naissance de l'écologie urbaine, Paris, Aubier, coll. " Champs urbains ", 1979).

10. Moi, Maurice, bottier à Belleville. Histoire d'une vie, récit recueilli par Michel Bloit, Paris, L'Harmattan, 1993, p. 80 et 81 . 
A L'ÉCHELle DU QUARTIER, UNE IMPLANTATION EN «NICHES ÉCONOMIQUES"

Petits commerçants et artisans participent au premier chef à la construction de l'identité d'un quartier, en particulier lorsqu'il s'agit d'un quartier d'immigration. Les étrangers acquièrent, dans les années 1920 et 1930, une place essentielle dans le milieu des commerçants et artisans bellevillois $^{11}$. Ils représentent déjà $15,8 \%$ des créateurs de petites entreprises en 1923-1925, mais plus du tiers en 1934-1936 (36,6\%) ${ }^{12}$. Le taux de renouvellement des petites entreprises patentées dans le bas-Belleville, entre 1925 et 1935 est de $80 \%$, en moyenne; il atteint près de $90 \%$ dans certains axes comme la rue Piat, la rue Lesage ou encore la rue Ramponeau, les mêmes où se groupent les petits entrepreneurs migrants. Pourtant, ce n'est que plus tard, dans les années 1950, que la présence étrangère s'impose dans les représentations comme l'une des caractéristiques de l'espace bellevillois.

En effet, les données recueillies sur le bas-Belleville témoignent de la pertinence d'une étude détaillée à l'échelle des rues: la répartition des étrangers par rues n'est pas homogène sur l'ensemble du quartier et l'on observe, notamment, des concentrations au nord de l'espace considéré (rue Piat et Villa Othoz, rue Jouy-Rouve, impasse de Gênes). Surtout, certains axes semblent singulièrement à l'écart de l'implantation étrangère: petites impasses dont la forte stabilité ne permet pas d'offrir d'espaces aux nouveaux arrivés (ainsi du passage Pékin, de la Cité de Gênes ou de la Cité Bisson dont nombre d'habitants recensés en 1926 sont encore présents en 1936) ou grand boulevard dont l'importance s'accompagne vraisemblablement d'une cherté des loyers qui le rend difficilement accessible pour des migrants, peu fortunés pour la plupart (boulevard de Belleville). Le critère national détermine également des localisations encore plus précises qui n'apparaissent qu'à la lumière d'une étude fine, par axe: les Polonais, largement majoritaires parmi les étrangers de Belleville $(68,4 \%)$, privilégient les axes pénétrants qui traversent le bas-Belleville du sud au nord, partant du boulevard de Belleville le plus souvent: la rue des Couronnes, la rue Ramponneau, la rue de Belleville. Mais ils délaissent singulièrement la rue Jouy-Rouve, lieu d'implan-

\footnotetext{
11. Les résultats qui suivent sont issus d'une étude sur le cas bellevillois: Claire Zalc, «Les petits entrepreneurs étrangers à Belleville entre les deux guerres", dans Natacha Coquery (sous la direction de), La boutique et la ville, Commerce, commerçants, espaces et clientèles, XVI ${ }^{e_{-}}$ $x x^{e}$ siècles, Tours, Publications de l'Université François-Rabelais de Tours, 2000, p. 403-421.

12. Gérard Jacquemet, "L'entreprise à Belleville dans l'entre-deux-guerres", Entreprises et entrepreneurs $\mathrm{XIX}^{e}-\mathrm{XX}^{e}$ siècles, congrès de l'Association française des historiens économistes, mars 1980 , p. 237.
} 
tation privilégié des Turcs et des Arméniens (qui représentent près de $60 \%$ des étrangers de la rue).

Préciser encore l'échelle d'observation entraîne des changements de perspective qui font apparaitre les logiques individuelles ou familiales de localisation des migrants. Ainsi, du côté impair de la rue des Couronnes (du numéro 1 au 57), on compte 94 Polonais; 65 d'entre eux se regroupent à trois adresses. Et si aucun des axes du quartier ne recense, en 1936, une majorité d'étrangers, dans les immeubles, de nombreuses adresses constituent des lieux de forte concentration de migrants. Le cosmopolitisme du 18 de la rue Denoyez qui, sur 145 occupants, recense 45 Polonais, 5 Roumains, 11 Arméniens, 4 Italiens, 5 Turcs, 2 Grecs, 3 Belges, un Libanais et un Luxembourgeois (soit plus de la moitié des étrangers présents dans la rue à la même date) contraste avec la composition des deux immeubles qui le suivent immédiatement: aux 16 et 14 de la même rue, les habitants recensés (respectivement 88 et 161 ) sont tous de nationalité française. Pourtant aucun hôtel ni garni n'est installé au 18 de la rue Denoyez ${ }^{13}$. Cet exemple témoigne des vertus du changement d'échelle pour comprendre les modalités d'implantation des migrants à Belleville. Les étrangers n'y sont pas majoritaires et le modèle du ghetto, tel qu'il est théorisé par l'École de Chicago dans les années vingt, ne convient pas pour décrire le quartier ${ }^{14}$. De plus, s'il est possible d'observer des micro-concentrations de migrants regroupés par nationalité ou par origine, elles ne s'accompagnent pas nécessairement d'un marquage professionnel spécifique du territoire.

L'étude des implantations commerciales et artisanales des étrangers à Belleville traduit, pour une part, le chevauchement entre espaces domestique et professionnel, au sein d'ateliers qui prennent place dans les appartements, ou de boutiques qui ouvrent sur la cuisine familiale. La dissociation entre habitat et activité économique n'est pas encore réalisée dans l'entre-deux-guerres, alors que les activités commerciales et artisanales sont bien souvent présentes au rez-de-chaussée des immeubles, mais également au fond des cours et dans les étages ${ }^{15}$. La pièce principale de

13. Archives de Paris, PER/292, Annuaire Didot-Bottin. Les volumes classés par rues sont disponibles pour les années 1919, 1929, 1931 et 1937.

14. Louis Wirth, Le ghetto, traduit par Pierre-Jacques Rojtman, Grenoble, Presses Universitaires de Grenoble, 1980.

15. Geoffrey Crossick et Heinz-Gerhard Haupt montrent l'interpénétration des espaces domestique et commercial au XIX ${ }^{\mathrm{e}}$ siècle: The petite Bourgeoisie in Europe 1870-1914, Enterprise, Family and Independence, Londres et New York, Routledge, 1995, p. 118 et suiv. Mais l'imbrication perdure dans l'entre-deux-guerres: Claire Levy-Vroelant, «Petit commerce et habitat entre 1930 et 1950 à Paris: de l'imbrication à la dissociation", dans N. Coquery (sous la direction de), $L a$ boutique et la ville..., op. cit., p. 423-445. 
l'appartement est consacrée à l'activité à laquelle participent tous les membres de la famille; l'installation dans un local spécifique, récompense de l'ascension économique, reste marginale dans les premiers temps qui suivent la migration. Cette imbrication est particulièrement forte dans la rue Piat et la rue Jouy-Rouve, espaces de concentration des ateliers de chaussures : en effet, le secteur des cuirs et peaux bénéficie, dans les années 1920, de l'arrivée de très nombreux artisans étrangers qui se lancent dans la fabrication et la vente de chaussures, mais également, en amont, dans la vente des peaux, crépins, boucles et semelles et, en aval, dans la réparation des chaussures ou encore dans les activités dérivées du secteur comme la maroquinerie. Le manque de visibilité des petits commerçants et artisans étrangers rappelle ainsi les modalités classiques de "niches économiques " étrangères fréquemment situées dans les interstices de l'espace urbain. Pourtant la relative invisibilité des petits entrepreneurs étrangers contraste avec la densité des réseaux interpersonnels qui les relient. Les modalités d'implantation des migrants indépendants dans l'agglomération parisienne ne sont que les projections, dans l'espace urbain, des toiles tissées par les filières migratoires.

\section{Inscriptions spatiales des filières migratoires}

Avant tout, il faut rappeler qu'un certain nombre de contraintes, économiques notamment, conditionnent l'indépendance professionnelle. Les liens d'interconnaissance jouent ainsi un rôle moteur dans l'exercice de l'activité commerçante et artisanale; ils se déclinent selon plusieurs modalités : couple, réseau de parenté ou encore origine commune. Les territoires des petits entrepreneurs paraissent ainsi épouser les formes modelées par les réseaux familiaux, amicaux ou encore migratoires dans lesquels ils s'inscrivent.

\section{LES ESPACES DE LA PARENTÉ}

Ces réseaux s'inscrivent tout d'abord dans les sphères de la parenté restreinte au couple et à ses enfants. Le partage des locaux correspond à la modalité la plus simple d'inscription spatiale des réseaux de la migration; il s'articule avec la fréquente dimension familiale de l'activité indépendante. La contribution du conjoint à l'activité de la petite entreprise est très souvent essentielle, qu'il tienne la caisse, fasse les comptes ou occupe une place dans l'atelier: «les trois quart des couples travaillaient ensemble parce que le mari avait besoin d'une finisseuse, pour coudre les boutons, 
pour faire les "sur-passages". Il arrivait que, quand mon père était à la machine, c'était ma mère qui allait livrer" se souvient Étienne Raczymow dont les parents, venus de Pologne, s'installent comme giletiers à Belleville dans les années $1920^{16}$. Après quelques années de travail à domicile, le père ouvre un atelier au 43 rue Piat où il emploie les membres de la famille nouvellement arrivés dans le quartier, avant qu'ils soient en mesure, euxmêmes, de "s'installer à leur compte». La co-résidence ne touche pas uniquement le conjoint ou les enfants du petit entrepreneur; elle concerne également les membres de réseaux de parenté plus distendus.

On a pu ainsi observer, à partir d'une étude sur les petits commerçants italiens, le cas de logements servant d'adresse à plusieurs entrepreneurs : la même adresse, à Pantin, voit par exemple se succéder cinq petits commerçants italiens portant le même patronyme, entre 1922 et 1930, tous spécialisés dans le commerce de fruits et dont trois sont nés dans le même village en Italie $^{17}$. La co-résidence permet alors d'expliquer la rapidité des rythmes d'installation de certains petits commerçants et artisans immigrants. Quelques jours seulement séparent parfois la date déclarée d'entrée en France et la date d'immatriculation au registre du commerce, le migrant disposant, dès son arrivée, des ressources matérielles (local, stock de marchandises) et immatérielles (informations, réseaux de fournisseurs et clientèles) de la filière migratoire.

Le mariage apparait également comme l'un des moyens d'accès à la boutique pour les étrangers. Ainsi, David Rostein, né en 1894 à Varsovie, est "brocanteur ambulant» du côté de La Villette lorsqu'il rencontre Louise Crance, divorcée, originaire de Nancy, qui tient depuis 1927 la boucherie "Maison Crance» du 23 rue Bisson, à Belleville. David, après avoir épousé Louise, passe du statut d'ambulant au statut de petit commerçant avec boutique puisqu'il immatricule à son nom, la boucherie de la rue Bisson en avril 1935 alors que Louise déclare cesser son activité de bouchère en se radiant du registre. Pendant la guerre, lorsque le gouvernement de Vichy interdit le commerce aux Juifs, Louise revient comme un prête nom: le 23 octobre 1941, David vient déclarer sa radiation du registre du commerce sous le motif suivant: "commerce passe à l'épouse». On note alors le rôle de la solidarité matrimoniale dans l'exercice de l'activité professionnelle. Cette stratégie permet à David de reprendre son activité après guerre: la boucherie échappe aux mesures d'aryanisation.

16. Belleville, Belleville, visages d'une planète, Paris, Créaphis, 1994, p. 58.

17. Judith Rainhorn et Claire Zalc, "Commerce à l'italienne? Activité professionnelle et immigration à Paris dans l'entre-deux-guerres ", Le Mouvement social, $\mathrm{n}^{\circ} 191$, avril-juin 2000, p. 49-68. 
Cet exemple permet surtout de montrer que les réseaux ne se limitent pas aux frontières du couple: en effet, une filière tant migratoire que professionnelle semble s'articuler autour de cette union entre David et Louise. Car on retrouve plusieurs bouchers portant un patronyme identique et qui s'installent dans le quartier dans les années qui suivent: Moszes Rostein, né en 1901 à Varsovie, ouvre en 1930 une boucherie rue des Amandiers, non loin de la rue Bisson. Quant à Léon Rostein, il s'installe également comme boucher trois ans plus tard, de l'autre côté de la rue de Belleville cette fois-ci, dans le $19^{\mathrm{e}}$. Sans compter les autres commerçants Rostein qui s'installent dans le quartier comme épiciers, bonnetiers et fourreurs. Bien que nos sources ne nous permettent pas d'établir avec certitude les liens de parenté entre ces trois individus, l'approche patronymique met en lumière la densité des réseaux de petits entrepreneurs.

Ces réseaux ne sont pas uniquement circonscrits à l'espace de la parentèle même élargie : l'origine commune semble également structurer filières migratoires et espaces d'installation de petits commerçants et d'artisans.

\section{LA PROXIMITÉ DES ORIGINES}

Ainsi, l'étude des lieux de naissance des petits entrepreneurs rend possible la reconstitution de réseaux professionnels articulés autour d'une origine commune. Les filières migratoires ne semblent pas circonscrites aux espaces de parenté, elles regroupent également les individus d'une même localité, voire d'une même origine. On retrouve ainsi quatre artisans du secteur de la chaussure, tous nés dans le même village de Turquie, qui ouvrent leurs ateliers rue Piat, à quelques mètres les uns des autres, dans les années 1920. Le premier installé se charge non seulement d'informer ses compatriotes sur les opportunités en locaux, en matériaux et en débouchés mais peut également prêter de l'argent ou participer à la constitution des stocks de marchandises.

Il est d'ailleurs possible de reconstituer plusieurs réseaux de petits commerçants étrangers, nés dans des communes identiques, et qui s'installent dans un périmètre urbain proche. Les Abramici, Opstein et les Carenfeld, tous nés à Kichineff, capitale de la Bessarabie, ouvrent leurs petites entreprises dans la même rue. Le réseau se structure alors autour de l'adresse des commerces sans pour autant être organisé autour de la même activité: l'un est horloger, l'autre boucher épicier et le troisième bijoutier. Pourtant, que ce soit sur les juifs du Pletzl, dans le quartier du Sentier ou sur les Italiens dans l'Est parisien, les travaux sur les communautés immigrées à Paris, tout en mettant en évidence l'existence d'espaces d'implantation privilégiés par les étrangers d'une même origine, ont 
montré que le modèle de la ségrégation ne fonctionnait pas pour décrire l'habitat immigré à Paris ${ }^{18}$. L'absence de concentration statique apparaît avec l'introduction, dans l'étude, des mobilités dans l'espace.

\section{ITINÉRAIRES DANS LA VILLE}

La source principale de cette étude, les registres d'immatriculation au registre du commerce, permet d'introduire une dimension dynamique dans l'étude spatiale en reconstituant les trajets urbains des petits entrepreneurs sur la période. En effet, l'existence d'un fichier alphabétique rend possible la recension de l'ensemble des immatriculations effectuées par un même individu et, par conséquence, de reconstituer à la fois son parcours professionnel et son itinéraire dans les limites du département de la Seine. De plus, les petits entrepreneurs sont théoriquement tenus de déclarer au greffe du tribunal de commerce leurs changements d'adresses professionnelles. Une étude a été menée afin de reconstituer les trajets urbains de 338 individus portant 76 patronymes distincts, afin de mieux comprendre le poids des filières migratoires dans les modalités d'inscription spatiale des petits entrepreneurs.

Les mobilités des petits entrepreneurs migrants dans l'agglomération, au cours de leur itinéraire professionnel, ne suivent pas la même direction selon qu'ils s'insèrent dans tel ou tel réseau d'interconnaissance. L'origine migratoire détermine ainsi, pour une part, le sens des trajets dans la ville des petits commerçants et artisans. Ainsi, la majorité des petits commerçants nés en province qui changent d'adresse, choisissent une seconde implantation dans les alentours immédiats de leur adresse précédente: $53 \%$ d'entre eux ne quittent pas l'arrondissement ou la commune de banlieue où il sont établis. Quant aux $47 \%$ restants, ils se dirigent pour la plupart vers la périphérie (60\%), s'éloignant du centre de la capitale. À titre d'illustration, les itinéraires urbains des cinq commerçants nommés Sanaux immatriculés au registre du commerce de la Seine, qui sont tous originaires du village de Ledergues, dans l'Aveyron, suivent la même direction: partis des Lilas dans les années 1920, ils s'installent au Perreux puis à Bonneuil. Les mobilités urbaines empruntent ainsi des trajets souvent déterminés par le réseau d'interconnaissance au sein duquel le petit entrepreneur s'inscrit tant professionnellement que socialement.

18. Voir Nancy Green, Les Travailleurs immigrés juifs à la Belle Époque: le «Pletzl» à Paris, Paris, Fayard, 1985 et Du Sentier à la Septième Avenue. La confection et les immigrés, Paris-New York, 1880-1980, Paris, Seuil, 1998; Marie-Claude Blanc-Chaléard, Les Italiens dans l'Est parisien. Une histoire d'intégration (1880-1960), Rome, collection de l'École française de Rome, $\mathrm{n}^{\circ}$ 264, 2000 . 
La comparaison entre les différents migrants paraît, là encore, éclairante. Si la noria migratoire entraîne les petits commerçants italiens de La Villette vers les communes de banlieue limitrophes, notamment à Pantin, Aubervilliers, Drancy ou La Courneuve, on observe, au contraire, un surprenant mouvement centripète des localisations des entrepreneurs juifs polonais des périphéries parisiennes, vers les arrondissements plus centraux de la capitale, notamment le $11^{\mathrm{e}}$, le $3^{\mathrm{e}}$ et le $4^{\mathrm{e}}$ arrondissements. De nombreux exemples attestent de la mobilité spatiale des petits entrepreneurs juifs polonais vers les centres plus anciennement constitués de la communauté. Or l'observation attentive de ces trajets laisse voir des logiques de déplacement déterminées par les solidarités déployées au sein de la filière migratoire. Leizor Orenstain, né en 1905 en Pologne s'installe en mai 1932 comme tailleur dans les périphéries bellevilloises, au 173 rue de Bagnolet dans le $2 \mathrm{O}^{\mathrm{e}}$ arrondissement. Il se rapproche progressivement du centre en immatriculant tout d'abord son atelier 43 rue Piat au mois de mai 1934, adresse où il est recensé en 1936. Son séjour à Belleville ne dure que quelques années et il trouve, en 1938, une localisation commerciale stable, 99 rue Oberkampf dans le $11^{\mathrm{e}}$ arrondissement. Cette stabilité s'accompagne d'un certain succès économique comme en témoigne l'adoption, la même année, d'une structure juridique en société réunissant la femme de Leizor, puis son fils après la guerre. Or l'établissement ne s'est pas fait au hasard : un Pisen Orenstain est recensé comme horloger au 80 de la rue Oberkampf depuis 1921. Cet exemple n'est pas un cas isolé; il illustre la mobilité spatiale des petits entrepreneurs immigrants: le territoire des juifs polonais s'étend ainsi vers le $11^{\mathrm{e}}$, puis le $4^{\mathrm{e}}$, comme pour se rapprocher de l'espace d'implantation originel de la communauté : le Pletzl ${ }^{19}$.

De plus, les trajets urbains n'indiquent pas forcément un déplacement des activités économiques: la domiciliation des petits entrepreneurs en banlieue concrétise, souvent, la dissociation progressive des espaces professionnel et domestique. Ainsi de ces quatre frères grecs, fabricants de chaussures, recensés à Belleville au début des années 1930 et dont l'adresse des domiciles coïncide avec l'adresse de l'entreprise : ils logent alors tous les quatre dans l'atelier. En 1935, le cadet quitte Paris pour la commune d'Arcueil; il est bientôt suivi par ses trois frères qui chacun, successivement, déclare une nouvelle adresse de domicile dans la banlieue sud. Mais la société de "vente et de fabrication de talons de bois" reste localisée dans la même rue bellevilloise, jusqu'en 1956. La réussite économique s'accompagne ici d'un départ vers la périphérie en même temps qu'elle consacre la dissociation des espaces domestique et professionnel. 
En effet, les solidarités activées au sein des filières migratoires ne rendent pas compte de l'ensemble des relations nouées, dans un quartier, autour de la petite entreprise. L'étude de l'insertion locale des petits commerçants et artisans étrangers doit également considérer les liens d'interconnaissance nés de l'échange marchand. Il ne faut pas négliger, non plus, les rapports de concurrence qui existent entre les différents petits entrepreneurs et qui peuvent, selon les contextes, prendre les chemins de la xénophobie.

\section{Solidarités et concurrences}

L'acte marchand donne lieu à des échanges de biens et de services, mais il suscite également des relations sociales spécifiques. Dans la boutique comme dans l'atelier, l'échange ne se réduit pas à sa dimension économique. La fonction d'intermédiaire ancre localement le petit entrepreneur qui participe de la formation sociale d'un territoire. Les liens qui se nouent autour des échanges économiques peuvent être l'occasion de solidarités mais également de concurrences, entre les migrants, et entre Français et étrangers.

SOLIDARITÉS IMMIGRÉES :

L'EXemple des boutiques D'Alimentation à Belleville

Les commerces d'alimentation, fréquentés quotidiennement par les habitants à une époque où l'approvisionnement en produits alimentaires s'effectue essentiellement dans les boutiques et sur les marchés, sont des lieux centraux de la sociabilité citadine: on se rencontre au café, mais aussi chez le boulanger ou l'épicier, pour converser, commérer, commenter; les boutiques d'alimentation se dotent de banquettes, de chaises, parfois de sofas ${ }^{20}$. Les ethnologues ont également montré le rôle joué par le secteur alimentaire dans la définition et la construction de l'«ethnicité». Les boutiques d'alimentation rendent possible la mise en place de pratiques communes de consommation et d'approvisionnement, favorisent la sociabilité et fonctionnent comme des marqueurs de l'insertion sociale et économique des populations migrantes dans les sociétés urbaines. À Chicago, dans les années 1920, les épiceries constituent de véritables institutions des communautés ethniques ${ }^{21}$. Qu'en est-il dans le Paris de l'entre-deux-guerres?

20. G. Crossick et H.-G. Haupt, The petite Bourgeoisie..., op. cit., p. 118.

21. Lizbeth Cohen, Making a New Deal. Industrial Workers in Chicago, 1919-1939, Cambridge, Cambridge University Press, 1990, p. 110 à 115 . 
À Belleville, si l'implantation des petits commerçants étrangers dans le secteur de l'alimentation ne correspond pas exactement à la cartographie des concentrations des domiciles des migrants, elle dessine le cœur du "quartier juif polonais» qui se forme progressivement dans les années 1920 et 1930, espace de référence de la communauté yiddishophone ${ }^{22}$. Alors que les seuls étrangers tenant commerce d'alimentation dans le bas-Belleville en 1925 sont belges et suisses, les Polonais et les Russes ouvrent, dans les années trente, épiceries, boulangeries et, surtout, boucheries. Marqueurs ethnico-religieux par excellence de la communauté juive, elles sont situées à proximité immédiate de la synagogue. Cependant, le centre de la communauté yiddish semble aussi constituer le centre du quartier immigrant dans son ensemble puisqu'à quelques mètres des boucheries polonaises, on trouve un épicier russe, un friturier suisse, un boulanger italien, un épicier arménien et deux fruitiers français, nés en Algérie. La perméabilité entre les différentes communautés transparait dans les sources qui dessinent la naissance d'un espace de "centralité immigrée", point nodal du quartier d'immigration en formation, lieu d'échange et de sociabilité partagé par les différentes nationalités ${ }^{23}$. Pourtant, même dans les espaces de forte concentration, les étrangers restent toujours minoritaires parmi les commerçants d'alimentation. Or, la place des étrangers dans l'univers de la boutique fait l'objet de contestations de plus en plus vives dans les années 1930.

\section{LA THÉMATIQUE DE LA CONCURRENCE ÉTRANGÈRE: \\ L'ÉPREUVE DE LA CRISE DES ANNÉES 1930}

Les contextes de difficultés économiques fournissent systématiquement le prétexte au développement de courants xénophobes dans la société française. Il est malaisé, au vu des sources disponibles, d'appréhender les modalités économiques du phénomène. Il faut se résoudre à lire la concurrence au travers des nombreuses productions imprimées des petits artisans et commerçants français. Or dès 1927 , lors de la crise inflationniste, un certain nombre d'organisations représentatives des commerçants se mobilisent contre la concurrence des étrangers. Des lettres sont envoyées au Ministère du Commerce s'inquiétant du "grand nombre d'étrangers provenant de l'Orient, qui viennent se fixer dans notre pays

22. Voir David Weinberg, Les Juifs à Paris de 1933 à 1939, Paris, Calmann-Lévy, 1974, notamment p. 22-23.

23. Je reprends l'expression de Jean-Claude Toubon et Khelifa Messamah, Centralité immigrée: le quartier de la Goutte d'Or: dynamique d'un espace pluri-ethnique: succession, compétition, cohabitation, Paris, L'Harmattan-CIEMI, 1990. 
pour y exercer des professions se rattachant au commerce des tissus et du cuir» en arguant "du nombre, déjà trop important, des commerçants étrangers de ce genre [qui] ne peut qu'aggraver les difficultés de commerçants français régulièrement établis et leur causer un sérieux préjudice ${ }^{24}$.

Pendant la crise des années 1930, alors que le petit commerce devient l'un des secteurs-refuges de l'emploi des immigrants, les textes de source professionnelle se font plus virulents contre les petits entrepreneurs étrangers. L'argument de la "concurrence déloyale» des étrangers devient un poncif des institutions représentatives de la profession. Ainsi de l'Association parisienne des Fabricants de Chapeaux pour dames qui déclare, en 1935, lutter "désespérément contre l'envahissement incessant de FAUX ARTISANS ÉTRANGERS \%. Il est difficile de mesurer les conséquences, en termes d'inscription urbaine, de la stigmatisation des étrangers dans les milieux commerçants. Néanmoins, la propension des étrangers à s'établir dans les axes à faible passage, ruelles ou impasses, s'accentue dans la seconde moitié de l'entre-deux-guerres. De même, l'étude des modes de gestion de l'étrangeté dans les mises en scène commerciales permet, par exemple, d'approcher les pratiques, croissantes dans les années 1930, de dissimulation de l'origine par le choix d'un nom de commerce qui "francise» le nom du commerçant. Mais ces perspectives de recherche doivent encore faire l'objet de développements.

Il semble néanmoins possible de montrer l'efficacité de la mobilisation des petits entrepreneurs français: relayées par la Chambre de commerce de Paris, leurs revendications aboutissent avec l'institution, en 1935, d'une carte d'artisan étranger suivie, en 1938, de la mise en place de la carte de commerçant étranger. Le décret-loi du 12 novembre 1938 stipule alors qu'il est interdit à tout étranger d'exercer sur le territoire français une profession commerciale ou industrielle sans posséder une carte d'identité spéciale portant la mention "commerçant» et délivrée par le préfet du département où l'étranger exerce son activité. La carte de commerçant étranger, en instaurant un contrôle administratif sur l'établissement d'un commerçant, limite grandement la liberté d'ouvrir une boutique.

L'étude de l'insertion urbaine des petits entrepreneurs étrangers contribue à expliquer comment les étrangers se répartissent dans la ville, au rythme des différentes étapes de leur parcours migratoire comme de leur itinéraire économique. Elle a montré combien les implantations urbaines des petits commerçants et artisans étrangers étaient déterminées 
par les configurations relationnelles dans lesquelles ils s'insèrent. Ainsi, les filières professionnelles constituées autour des petites entreprises participent à alimenter les flux des migrants qui viennent s'installer dans certains quartiers de l'agglomération parisienne. Mais les boutiques et les ateliers des étrangers sont également des lieux de sociabilité partagés par l'ensemble des Parisiens. Avec la crise, alors que le marché du travail salarié se ferme progressivement à la main d'œuvre étrangère, le petit commerce et l'artisanat s'instituent comme des secteurs-refuge. Dans les rues de la capitale, l'étrangeté devient alors un stigmate. 